\title{
Pulmonary Function Test Increased
}

National Cancer Institute

\section{Source}

National Cancer Institute. Pulmonary Function Test Increased. NCI Thesaurus. Code C121578.

An increase in any of the parameters used to measure the mechanical function of the lungs and associated structures. 